\title{
High expression of microRNA-208 is associated with cardiac hypertrophy via the negative regulation of the sex-determining region Y-box 6 protein
}

\author{
XINTAO HUANG ${ }^{1}$, ZHIHENG LI $^{2}$, BAOQIANG BAI ${ }^{1}$, XIAOHONG LI $^{1}$ and ZHONGYUAN LI ${ }^{1}$ \\ Departments of ${ }^{1}$ Cardiology and ${ }^{2}$ Neurology, Zhumadian Central Hospital, Zhumadian, Henan 463000, P.R. China
}

Received November 26, 2014; Accepted July 2, 2015

DOI: $10.3892 / \mathrm{etm} .2015 .2645$

\begin{abstract}
The aim of this study was to investigate the expression levels of microRNA-208 (miR-208) and sex-determining region Y-box 6 (SOX6) in patients with progressive cardiac hypertrophy. A total of 50 patients with essential hypertension accompanied by left ventricular hypertrophy, and 30 healthy individuals were enrolled. Peripheral blood samples were collected in order to compare miR-208 expression levels between the cardiac hypertrophy patients and healthy individuals. In addition, an in vitro cellular model of cardiac hypertrophy was established to determine the association between miR-208 and SOX6 expression. Rat cardiomyocytes were treated with phenylephrine (PE) to induce cardiac hypertrophy. Some of the hypertrophic cardiomyocytes were subsequently transfected with antagomiR-208, an miR-208 antagonist, in order to determine the effects of silencing miR-208 expression. Differences between healthy and hypertrophic cardiomyocyte morphology were evaluated using immunofluorescence staining. The mRNA expression levels of the hypertrophy-associated genes $\beta$-myosin heavy chain, $\alpha$-sarcomeric actin and atrial natriuretic peptide were determined using quantitative polymerase chain reaction (qPCR). The mRNA and protein expression levels of miR-208 and SOX6 in peripheral blood and cardiomyocytes were detected using qPCR and western blot analysis, respectively. The expression levels of miR-208 were significantly increased in the peripheral blood of patients with left ventricular hypertrophy $(\mathrm{P}<0.05)$. PE-stimulated cardiomyocytes were significantly increased in size compared with normal cardiomyocytes. In the PE-stimulated cardiomyocytes, miR-208 expression levels were significantly increased $(\mathrm{P}<0.05)$. However, SOX6 expression levels were significantly decreased compared with those in normal cardiomyocytes $(\mathrm{P}<0.05)$. Following transfection with
\end{abstract}

Correspondence to: Dr Xintao Huang, Department of Cardiology, Zhumadian Central Hospital, 747 Zhong Hua Road, Zhumadian, Henan 463000, P.R. China

E-mail: Hxt000@126.com

Key words: hypertrophy, microRNA-208, sex-determining region Y-box 6, cardiomyocytes
antagomiR-208, SOX6 expression levels in the PE-stimulated cardiomyocytes significantly increased, while the total mRNA and protein expression levels of hypertrophy-associated genes significantly decreased $(\mathrm{P}<0.05)$. miR-208 expression levels are increased in the peripheral blood of patients with cardiac hypertrophy. Therefore, the results of this study suggest that the expression levels of miR-208 are associated with cardiac hypertrophy by the negative regulation of SOX6.

\section{Introduction}

Essential hypertension is a common chronic disease that has no identifiable cause. Essential hypertension may increase the risk of coronary heart disease, stroke, kidney failure and heart failure, all of which may be seriously harmful to human health (1). In recent years, due to progressively aging populations, improved living standards and stress, the incidence of essential hypertension has increased significantly (2). The prevalence of essential hypertension in young patients is increasing, and there is thus an urgent requirement for methods to prevent and treat essential hypertension.

Cardiac hypertrophy is a common pathological manifestation in patients with essential hypertension, which is primarily caused by prolonged cardiac stress. The myocardium thickens in order to compensate for the increase in blood pressure, eventually leading to heart failure (3). A previous study demonstrated that gene expression profiles in cardiac mast cells differ significantly between patients with cardiac hypertrophy and healthy donors, and cardiac hypertrophy is reversible in the early stages (4). Therefore, elucidation of the molecular mechanisms of essential hypertension accompanied by cardiac hypertrophy is of clinical significance in the diagnosis and treatment of hypertrophic cardiomyopathy.

microRNA (miRNA) is a type of small endogenic non-coding RNA molecule (18-22 nucleotides) that regulates gene expression by binding to 3'-untranslated regions of target mRNA. miRNA molecules are widely present in the tissues and blood, with good stability. The expression levels of various miRNA in cardiac mast cells exhibit significant alterations in cardiac hypertrophy, which suggests that miRNAs serve key functions in the progression of cardiac hypertrophy $(5,6)$. miRNA-208 is highly expressed in the serum of patients with myocardial infarction, which indicates that miR-208 may be a suitable biomarker for the diagnosis of myocardial damage (7). 
Oliveira-Carvalho et al reported a strong association of miR-208 expression in cardiac mast cells with hypertrophic cardiomyopathy, suggesting that miR-208 may be involved in the progression of the condition, although the mechanism remains unclear (8). miR-208 promotes the proliferation of esophageal squamous cell carcinoma cells by regulating the gene sex-determining region Y-box 6 (SOX6) (9). The SOX gene family encodes a group of transcription factors defined by the conserved high mobility group DNA-binding domain, which is closely associated with a variety of types of cell differentiation and embryonic development $(10,11)$. However, the role of SOX6 in hypertrophic cardiomyopathy remains unknown. In the present study, the expression levels of miR-208 and SOX6 in the peripheral blood of patients with essential hypertension accompanied by left ventricular hypertrophy were determined by western blot analysis and quantitative polymerase chain reaction (qPCR) assay. Furthermore, the mechanism underlying the affects of miR-208 in the progression of cardiac hypertrophy were further studied.

\section{Materials and methods}

Reagents. TRIzol was purchased from Invitrogen Life Technologies (Carlsbad, CA, USA). Takara PrimeScript RT Reagent kit and SYBR PrimeScript RT-PCR kit II (Perfect Real Time) were purchased from Takara Bio, Inc. (Otsu, Japan). Rabbit anti-rat SOX6 polyclonal antibodies (sc-20092), rabbit anti-rat $\alpha$-sarcomeric actin ( $\alpha$-SA) monoclonal antibodies (sc-135072) and DAPI were purchased from Santa Cruz Biotechnology, Inc. (Santa Cruz, CA, USA). Rabbit anti-rat glyceraldehyde 3-phosphate dehydrogenase (GAPDH; $\mathrm{MB} 001 \mathrm{H})$ monoclonal antibodies and horseradish peroxidase (HRP)-conjugated goat anti-rabbit antibodies were purchased from Bioworld Technology, Inc. (BS1327; St. Louis Park, MN, USA). High-glucose Dulbecco's modified Eagle's medium (H-DMEM) and fetal bovine serum (FBS) were purchased from Gibco Life Technologies (Carlsbad, CA, USA). Phenylephrine (PE) was from Sigma-Aldrich (St. Louis, MO, USA) and antagomiR-208 was from Guangzhou RiboBio Co., Ltd. (Guangzhou, China).

Clinical data. A total of consecutive 50 patients with essential hypertension were enrolled in this study. All patients had left ventricular hypertrophy diagnosed by ultrasound. The patient population with essential hypertension included 31 men and 19 women, aged between 17 and 66 years, with an average age of 63.4 years. Duration of illness in these patients was $>5$ years. A total of 30 healthy individuals were enrolled in this study consecutively as a control group. Peripheral blood was collected from the patients and healthy individuals. Prior written and informed consent were obtained from each patient. The study was approved by the ethics review board of Zhumadian Central Hospital (Zhumadia, China).

Cell culture and transfection. Primary cultures of neonatal rat cardiomyocytes were established according to a procedure published previously (12). Briefly, hearts excised from neonatal Sprague-Dawley rats (Sibeifu Beijing Laboratory Animal Science and Technology Co., Ltd., Beijing, China) were cut into $1.0-\mathrm{mm}$ pieces and digested in phosphate-buff-
Table I. Primer sequences.

\begin{tabular}{ll}
\hline Gene & \multicolumn{1}{c}{ Sequences (5'-3') } \\
\hline miR-208 & F: 5'-CTTTTGGCCCGGGTTATAC-3' \\
SOX6 & R: 5'-CTGACATCCTCTAGGCTGG-3' \\
& F: 5'-GTCATCCAGAGCACTTAT-3' \\
U6 & R: 5'-TCTAAAGACTGGAAGGAG-3' \\
& F: 5'-CTCGCTTCGGCAGCACA-3' \\
$\beta$-MHC & R: 5'-AACGCTTCACGAATTTGCGT-3' \\
& F: 5'-GACAGATGAAGACGATGAC-3' \\
$\alpha$-SA & R: 5'-TGATGCTCCAAGGTAACT-3' \\
& F: 5'-GTAACAACAGCATAAGAA-3' \\
ANP & R: 5'-TTACAGGTATTACAGAGAA-3' \\
& F: 5'-TACGCCTTCTTCAACATT-3' \\
& F: 5'-ACGAGTATGCTTGCTTAG-3'
\end{tabular}

miR, microRNA; SOX6, sex-determining region Y-box 6; $\beta$-MHC, $\beta$-myosin heavy chain; $\alpha$-SA, $\alpha$-sarcomeric actin; ANP, atrial natriuretic peptide.

ered saline (PBS) with $0.5 \%$ collagenase II and trypsin (Gibco Life Technologies) for $5 \mathrm{~min}$ at $37^{\circ} \mathrm{C}$. The supernatant of the heart tissue digests was transferred to a new tube, and the digestion was repeated twice. The heart tissue digests were suspended in H-DMEM supplemented with $10 \%$ FBS. The cardiomyocytes in suspension were collected and seeded in a 25-ml culture flask containing H-DMEM supplemented with $10 \%$ FBS. Cells were incubated at $37^{\circ} \mathrm{C}$ in $5 \% \mathrm{CO}_{2}$. In order to establish an in vitro cellular model of cardiac hypertrophy, $60 \%$ confluent primary cardiomyocytes were treated with $\mathrm{PE}$ at a final concentration of $100 \mu \mathrm{M}$ for $72 \mathrm{~h}$ in serum-free H-DMEM. After a 48-h culture, the establishment of a cellular model of cardiac hypertrophy was confirmed by bicinchoninic acid assay (Biyuntian, Beijing, China) and expression of hypertrophy associated genes, including $\beta$-myosin heavy chain ( $\beta$-MHC), $\alpha$-SA and atrial natriuretic peptide (ANP).

Transfection with antagomiR-208 was performed in 70\% confluent cardiomyocytes. Prior to transfection, cardiomyocyte in the logarithmic phase were transferred to a 24-well plate and incubated in serum-free H-DMEM at $37^{\circ} \mathrm{C}$ in $5 \% \mathrm{CO}_{2}$ for $24 \mathrm{~h}$. The medium was replaced with $200 \mu \mathrm{l}$ serum-free RPMI-1640 medium (Gibco Life Technologies) mixed with $4 \mu \mathrm{l}$ antagomiR-208 (40 pmol $/ \mu \mathrm{l})$. Analyses were performed at $72 \mathrm{~h}$ after transfection. An miR-208 independent sequence was used as negative control.

Reverse transcription (RT)-qPCR assay. Total RNA was extracted from peripheral blood samples and cardiomyocytes using TRIzol reagent. RNA was quantified using electrophoresis and spectrophotometric determination of the 260/280 nm optical density ratio. All RNA was reverse transcribed into cDNA with PrimeScript RT Reagent kits. qPCR was conducted using SYBR PrimeScript RT-PCR kit II (Perfect Real Time) in accordance with the manufacturer's instructions. U6 was used as an internal control against which miR-208, SOX6, $\beta$-MHC, $\alpha$-SA and ANP were compared. The oligonucleotide primers 
A

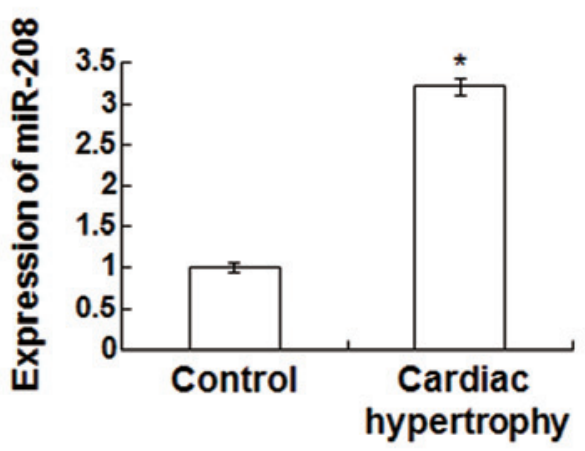

B

\section{Normal cardiomyocytes}

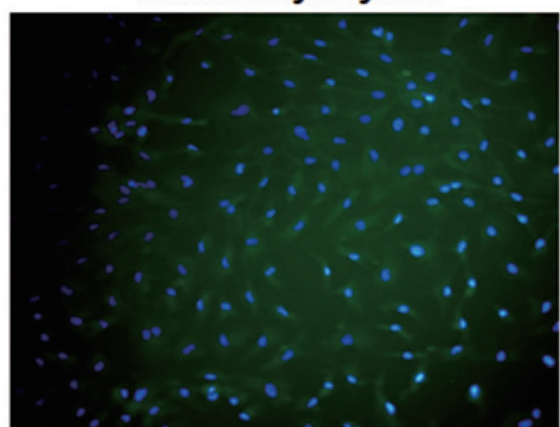

C

\section{PE-stimulated cardiomyocytes}

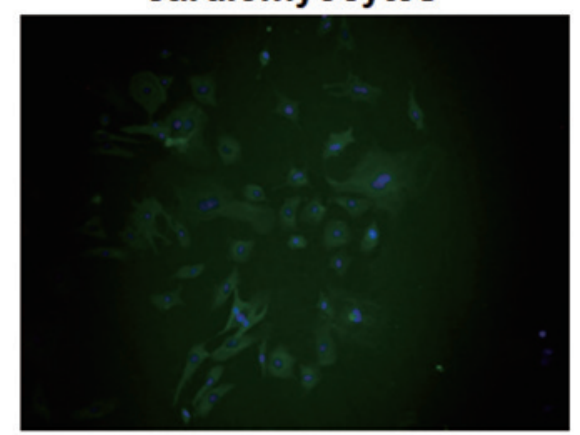

Figure 1. Expression levels of miR-208 in the peripheral blood were detected using quantitative polymerase chain reaction assays, and the morphology of the cardiomyocytes was observed using immunofluorescence staining. (A) The expression levels of miR-208 were determined and the experiments were repeated at least three times. Bar graphs showed the mean normalized expression levels of miR-208 in the peripheral blood of patients with left ventricular hypertrophy and healthy individuals. Error bars show the standard error of the mean ( $\mathrm{P}<0.05$ vs. control). Fields of (B) the normal and (C) PE-stimulated cardiomyocytes were stained using immunofluorescence staining and visualized using an inverted fluorescence microscope (magnification, x200). The cytoplasm was stained in green, and nuclei were stained in blue.

used (Table I) were synthesized by Shanghai Invitrogen Biotechnology Co., Ltd. (Shanghai, China). For each sample, the $\mathrm{qPCR}$ assay was repeated at least three times.

Immunofluorescence staining. Cardiomyocytes cultured on coverslips were fixed with acetone for $1 \mathrm{~h}$ and washed with deionized $\mathrm{H}_{2} \mathrm{O}$ for $5 \mathrm{~min}$ at room temperature, which was repeated three times. In order to inactivate endogenous peroxidase, $3 \%$ fresh prepared hydrogen peroxide was added and the cardiomyocytes were incubated at room temperature for $20 \mathrm{~min}$. After washing with PBS, the coverslips were blocked in $5 \%$ bovine serum albumin for $1 \mathrm{~h}$. The primary anti- $\alpha-\mathrm{SA}$ antibodies (1:200) were added and the cardiomyocytes were incubated in the dark overnight at $4^{\circ} \mathrm{C}$. After rinsing, secondary fluorescein isothiocyanate-conjugated rabbit anti-rat IgG antibodies (1:200; Abcam, Cambridge, MA, USA) were added and the cardiomyocytes were incubated for $30 \mathrm{~min}$ at $37^{\circ} \mathrm{C}$. After staining the nucleus with DAPI for $5 \mathrm{~min}$, the coverslips were visualized under an IX83 inverted fluorescence microscope (Olympus Corporation, Tokyo, Japan) at high magnification (x200).

Western blot analysis. Cardiomyocytes were homogenized in radioimmunoprecipitation assay buffer. Total proteins were separated on $12 \%$ SDS-PAGE gels and then analyzed by immunoblot analysis. GAPDH was used as an internal control. The primary polyclonal rabbit anti-rat SOX6 $(1: 1,000)$ and anti-rat GAPDH antibodies $(1: 5,000)$ were used. The secondary antibodies used were HRP-conjugated goat anti-rabbit antibodies $(1: 1,000)$ and goat anti-rat antibodies $(1: 3,000)$. The western blots were visualized using BeyoECL Plus reagent (Beyotime Institute of Biotechnology, Haimen, China). Image quantification was performed using Quantity One software (Bio-Rad Laboratories, Inc., Hercules, CA, USA). The experiments were repeated at least three times.

Statistical analysis. All results are expressed as the mean \pm standard deviation. All statistical analyses were performed using SPSS software for Windows, version 11.0
(SPSS Inc., Chicago, IL, USA). Paired t-test was used to analyze comparisons between groups and analysis of paired data. $\mathrm{P}<0.05$ was considered to indicate a statistically significantly difference.

\section{Results}

Expression levels of miR-208 are increased in the peripheral blood of patients with left ventricular hypertrophy. In order to detect the expression levels of miR-208 in the peripheral blood of patients with left ventricular hypertrophy and healthy individuals, qPCR assays were performed. As shown in Fig. 1A, the expression levels of miR-208 in the peripheral blood of patients with left ventricular hypertrophy were $\sim 3.21 \pm 0.1$-fold higher compared with those of healthy individuals $(\mathrm{P}<0.05)$. Therefore, the qPCR results indicate that expression levels of miR-208 are increased in the peripheral blood of patients with left ventricular hypertrophy.

PE-stimulated cardiomyocytes are significantly larger than normal cardiomyocytes. In order to determine the morphological differences between the cellular model of cardiac hypertrophy and normal cardiomyocytes, immunofluorescence staining was performed. As shown in Fig. 1B, primary neonatal rat cardiomyocytes exhibited a typical polygon-shape and rod-shape. However, following stimulation with $100 \mu \mathrm{M}$ PE for $72 \mathrm{~h}$ (Fig. 1C), cardiomyocytes was significantly larger compared with the normal cardiomyocytes that did not receive PE treatment. These results show that the PE-stimulated cardiomyocytes in the cellular model of cardiac hypertrophy were significantly larger than normal cardiomyocytes.

Expression levels of hypertrophy-associated genes are increased in the PE-stimulated cardiomyocytes. In order to determine the expression levels of hypertrophy-associated genes in a cellular model of cardiac hypertrophy, qPCR assays were performed. Total proteins were detected in the PE-stimulated and normal cardiomyocytes using a bicinchoninic acid assay. As shown in Fig. 2A, the total protein 
A

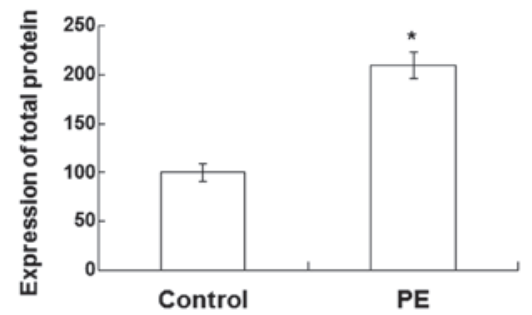

C

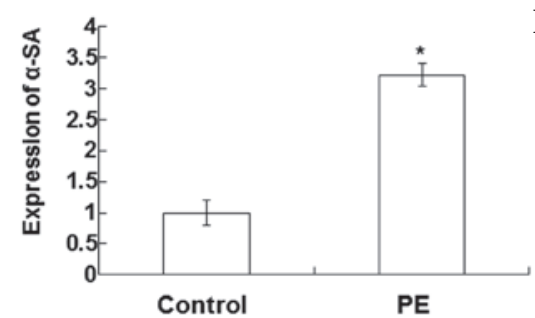

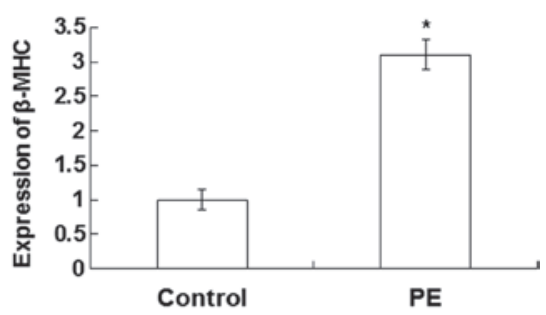

D

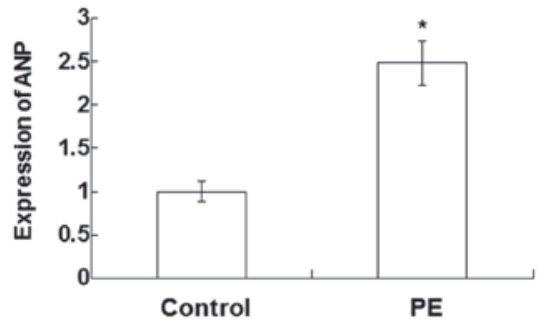

Figure 2. Total protein and mRNA expression levels of hypertrophy-associated genes in the normal and PE-stimulated cardiomyocytes. The total proteins in the normal and PE-stimulated cardiomyocytes were detected by bicinchoninic acid assay. The expression levels of hypertrophy-associated genes were detected using quantitative polymerase chain reaction. Bar graphs show (A) the mean normalized total protein, in addition to the mRNA expression levels of (B) $\beta$-MHC, (C) $\alpha$-SA and (D) ANP in the normal and PE-stimulated cardiomyocytes. Error bars show the standard error of the mean ("P<0.05 vs. control). All experiments were repeated at least three times. $\beta$-MHC, $\beta$-myosin heavy chain; PE, phenylephrine; $\alpha$-SA, $\alpha$-sarcomeric actin; ANP, atrial natriuretic peptide.

A

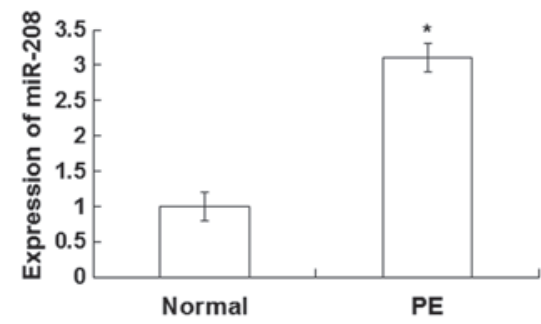

C

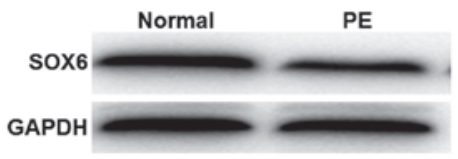

B

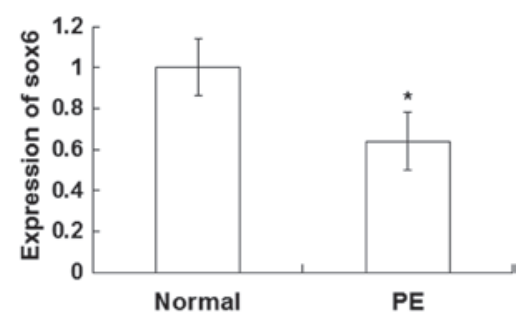

D

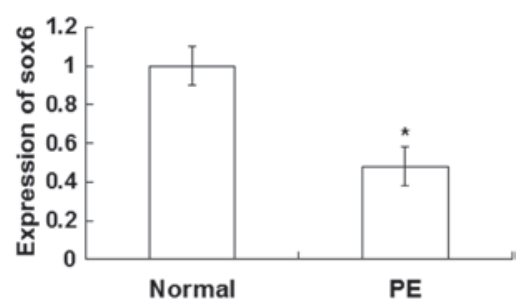

Figure 3. Expression of miR-208 and SOX6 in the normal and PE-stimulated cardiomyocytes. Expression levels of miR-208 and SOX6 were determined using quantitative polymerase chain reaction assay and western blot analysis. Experiments were repeated at least three times. Histograms show the mean normalized mRNA expression levels of (A) miR-208 and (B) SOX6 in the normal and PE-stimulated cardiomyocytes. For the detection of SOX6 protein expression, total proteins from the normal and PE-stimulated cardiomyocytes were collected and separated on 12\% SDS-PAGE gels, then analyzed by western blot. GAPDH was used as a loading control. (C) Representative and (D) quantitative western blot results. Error bars show the standard error of the mean ( $\mathrm{P}<0.05)$. miR-208, microRNA-208; PE, phenylephrine; SOX6, sex-determining region Y-box 6; GAPDH, glyceraldehyde 3-phosphate dehydrogenase.

levels in the PE-stimulated cardiomyocytes were significantly increased compared with those in normal cardiomyocytes $(\mathrm{P}<0.05)$. The qPCR results showed that the expression levels of $\beta$-MHC (Fig. 2B), $\alpha$-SA (Fig. 2C) and ANP (Fig. 2D) in the PE-stimulated cardiomyocytes were significantly increased compared with those in the normal cardiomyocytes $(\mathrm{P}<0.05)$. These results indicate that the expression levels of hypertrophy-associated genes are increased in the PE-stimulated cardiomyocytes.

miR-208 expression levels are increased, while SOX6 expression levels are decreased, in the PE-stimulated cardiomyocytes.
In order to investigate the expression levels of miR-208 and SOX6 in the PE-stimulated cardiomyocytes and normal cardiomyocytes, qPCR and western blot assays were performed. The qPCR results showed that miR-208 expression levels were significantly increased $(4.23 \pm 0.21$-fold $)$ in the PE-stimulated cardiomyocytes compared with those in the normal cardiomyocytes $(\mathrm{P}<0.05$; Fig. 3A). However, SOX6 expression levels were significantly decreased $(0.68 \pm 0.17$-fold $)$ in the PE-stimulated cardiomyocytes compared with those in the normal cardiomyocytes $(\mathrm{P}<0.05$; Fig. 3B). As shown by the western blotting results in Fig. 3C, the expression levels of SOX6 were significantly reduced in the PE-stimulated cardiomyocytes compared 
A

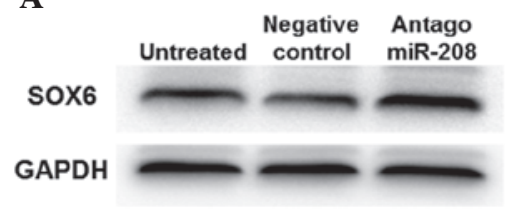

B

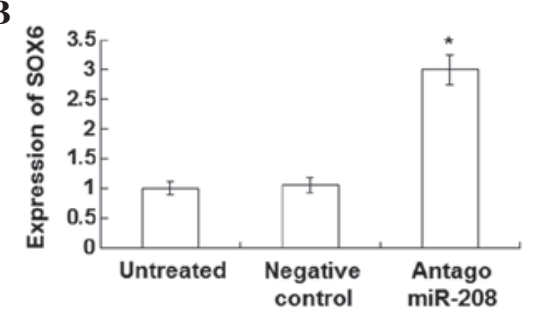

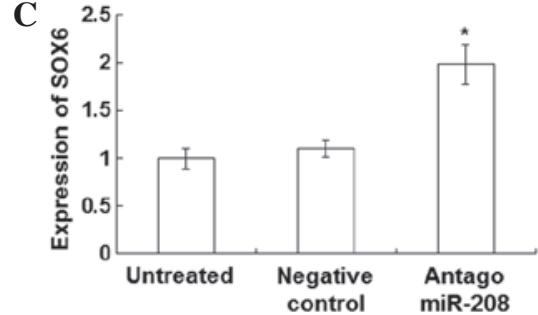

Figure 4. Expression of SOX6 in the normal and phenylephrine (PE)-stimulated cardiomyocytes following transfection with antagomiR-208. The expression levels of SOX6 were detected using western blotting and quantitative polymerase chain reaction (qPCR) assay at $72 \mathrm{~h}$ after transfection with antagomiR-208. An miR-208 independent sequence was used as a negative control. For the detection of SOX6 expression by western blotting, total proteins of the PE-stimulated cardiomyocytes transfected with antagomiR-208, the negative control and the PE-stimulated cardiomyocytes were collected and separated on 12\% SDS-PAGE gels. GAPDH was used as a loading control. (A) Representative and (B) quantitative western blot analysis results. Error bars show the standard error of the mean ("P<0.05 vs. negative control). Experiments were repeated at least three times. (C) Bar graph showing qPCR results of SOX6 expression levels in the PE-stimulated cardiomyocytes transfected with antagomiR-208, the negative control and the untransfected PE-stimulated cardiomyocytes. miR-208, microRNA-208; SOX6, sex-determining region Y-box 6; GAPDH, glyceraldehyde 3-phosphate dehydrogenase.
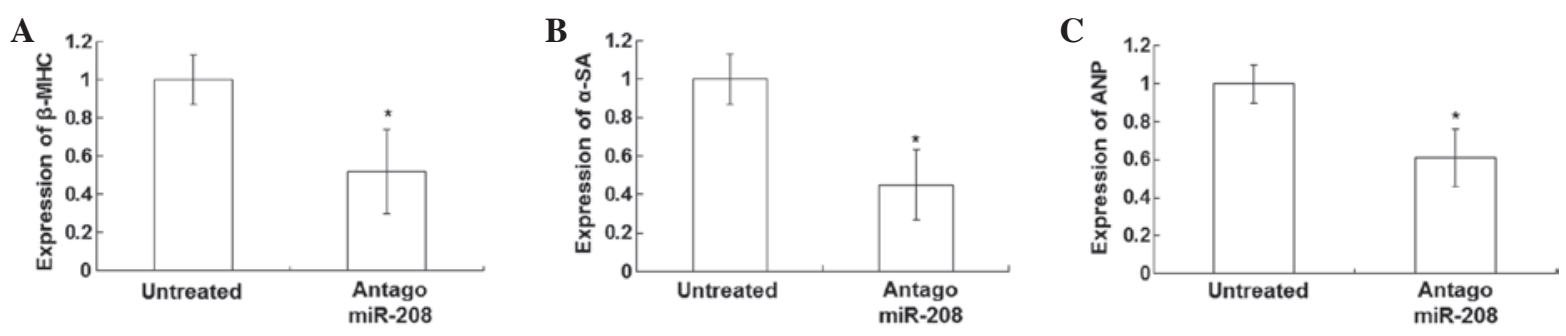

Figure 5. Expression of hypertrophy-associated genes in the phenylephrine (PE)-stimulated cardiomyocytes transfected with antagomiR-208 and the untransfected PE-stimulated cardiomyocytes. The expression levels of hypertrophy-associated genes were detected by quantitative polymerase chain reaction assay. Experiments were repeated at least three times. Bar graphs show the mean normalized mRNA expression levels of (A) $\beta$-MHC, (B) $\alpha$-SA and (C) ANP in the PE-stimulated cardiomyocytes transfected with antagomiR-208 and the untransfected PE-stimulated cardiomyocytes. Error bars show the standard error of the mean ("P<0.05 vs. untreated). $\beta$-MHC, $\beta$-myosin heavy chain; miR-208, microRNA-208; $\alpha$-SA, $\alpha$-sarcomeric actin; ANP, atrial natriuretic peptide.

with those in the normal cardiomyocytes. Relative values indicated that the quantity of SOX6 expression levels in the PE-stimulated cardiomyocytes were $\sim 0.48 \pm 0$.11-fold lower compared with those in the normal cardiomyocytes $(\mathrm{P}<0.05$; Fig. 3D). These results suggest that miR-208 expression levels are increased, whereas SOX6 expression levels are decreased in the PE-stimulated cardiomyocytes.

miR-208 is associated with cardiac hypertrophy by negative regulation of SOX6. In order to investigate the association between the expression levels of miR-208 and SOX6, transfection of the PE-stimulated cardiomyocytes with antagomiR-208 was performed. The mRNA and protein expression levels of miR-208 and SOX6 were detected using qPCR and western blot analysis, respectively. As shown in Fig. 4A, following transfection with antagomiR-208 in the PE-stimulated cardiomyocytes, SOX6 expression levels were significantly increased compared with those in untransfected PE-stimulated cardiomyocytes and negative control cells. The results of the quantitative western blot analysis are presented in Fig. 4B. The expression levels of SOX6, detected by qPCR assay, were significantly increased in the antagomiR-208-transfected cardiomyocytes compared with those of the untransfected PE-stimulated and negative control cardiomyocytes (Fig. 4C). Following transfection of the PE-stimulated cardiomyocytes with antagomiR-208, the total proteins were significantly decreased (data not shown). The qPCR results showed that the expression levels of $\beta$-MHC (Fig. 5A), $\alpha$-SA (Fig. 5B) and ANP (Fig. 5C) in the PE-stimulated cardiomyocytes after transfection with antagomiR-208 were significantly decreased compared with those without transfection $(\mathrm{P}<0.05)$.

These results indicate that the inhibition of miR-208 resulted in increased expression levels of SOX6. Therefore, miR-208 may be associated with cardiac hypertrophy, mediated by the negative regulation of SOX6.

\section{Discussion}

Cardiac hypertrophy is associated with the expression of various genes. Expression of the transcription factor myocardin may be inhibited by nuclear factor $\kappa$-light-chain-enhancer of activated B cells, which modifies the gene expression profile in cardiomyocytes, thereby inhibiting cardiac hypertrophy (13). Endothelin-1 is able to promote cardiac hypertrophy via the upregulation of ANP (14). The stimulation of transient receptor potential cation channel subfamily $\mathrm{V}$ member 1 can activate the PPAR- $\sigma$ signaling pathway, which plays an important role in inhibiting cardiac hypertrophy. Therefore, identification of new hypertrophy-associated genes and their underlying molecular mechanisms has clinical significance for the diagnosis and treatment of patients with essential hypertension accompanied with cardiac hypertrophy.

In cardiac hypertrophy, the expression of various miRNAs in cardiac mast cells exhibit significant differences from those in healthy individuals, which involve a range of signal 
pathways. Increased expression of miR-1 significantly inhibits cardiac hypertrophy via the regulation of connexin 43 (15). In addition, adrenaline and endothelin-induced cardiac hypertrophy can be inhibited by miR-133 in vitro. The expression of miR-133 is significantly decreased in cardiac mast cells, which suggests that miR-133 is closely associated with the development of cardiac hypertrophy. Recent studies have indicated that miR-423, miR-34, miR-190 and other miRNA molecules may be involved in the development of cardiac hypertrophy $(16,17)$.

In the present study, immunofluorescence staining, western blot analysis and qPCR assays were performed to evaluate the expression levels of miR-208 and SOX6 and their effects on the progression of cardiac hypertrophy. The association between the expression levels of miR-208 and SOX6 was investigated in a cellular model of cardiac hypertrophy, and by transfecting a proportion of the PE-treated rat cardiomyocytes with antagomiR-208. The regulatory mechanism of miR-208 was further studied. The present results showed that the expression levels of miR-208 were significantly increased in the peripheral blood of patients with left ventricular hypertrophy compared with those of healthy individuals $(\mathrm{P}<0.05)$. The PE-stimulated cardiomyocytes were significantly larger than normal cardiomyocytes. The expression levels of hypertrophy related genes, including $\beta$-MHC, $\alpha$-SA and ANP, were increased in the PE-stimulated cardiomyocytes $(\mathrm{P}<0.05)$. The qPCR results showed that the miR-208 expression levels were significantly increased in the PE-stimulated cardiomyocytes compared with normal cardiomyocytes $(\mathrm{P}<0.05)$. However, SOX6 expression levels were significantly decreased in the PE-stimulated cardiomyocytes compared with the normal cardiomyocytes $(\mathrm{P}<0.05)$. In the PE-stimulated cardiomyocytes that were transfected with antagomiR-208, the SOX6 expression levels were significantly increased. In addition, the total proteins and the mRNA expression levels of hypertrophy-associated genes were significantly decreased, which indicated that cardiac hypertrophy may be reduced by inhibiting miR-208 by transfection with antagomiR-208.

In conclusion, miR-208 expression levels are increased in the peripheral blood of patients with cardiac hypertrophy. The expression levels of miR-208 are associated with cardiac hypertrophy by negative regulation of SOX6. These results suggest that miR-208 may be useful as a novel therapeutic and diagnostic marker for cardiac hypertrophy.

\section{Acknowledgements}

The authors thanks Professor Xuemin Pang at the Department of Cardiology, Zhumadian Central Hospital (Zhumadian, China) for support.

\section{References}

1. Milouk FZ, El Bakkali M, Coghlan L, Lachhab A, Aboudrar S and Benjelloun $\mathrm{H}$ : Kinetics of orthostatic blood pressure in primary hypertension. Int Cardiovasc Res J 8: 83-88, 2014.
2. Prkačin I, Balenovic D, Cavrić G, Bartolek D and Bulum T: Importance of standardized stepwise screening in patients with resistant hypertension. Acta Med Croatica 68: 111-115, 2014 (In Croatian).

3. Shah AS, Chin CW, Vassiliou V, Cowell SJ, Doris M, Kwok TC, Semple S, Zamvar V, White AC, McKillop G, et al: Left ventricular hypertrophy with strain and aortic stenosis. Circulation 130: 1607-1616, 2014.

4. Kuster DW, Mulders J, Ten Cate FJ, Michels M, Dos Remedios CG, da Costa Martins PA, van der Velden J and Oudejans CB: MicroRNA transcriptome profiling in cardiac tissue of hypertrophic cardiomyopathy patients with MYBPC3 mutations. J Mol Cell Cardiol 65: 59-66, 2013.

5. Bernardo BC, Gao XM, Tham YK, Kiriazis H, Winbanks CE, Ooi JY, Boey EJ, Obad S, Kauppinen S, Gregorevic P, et al: Silencing of miR-34a attenuates cardiac dysfunction in a setting of moderate, but not severe, hypertrophic cardiomyopathy. PLoS One 9: e90337, 2014.

6. Hernandez-Torres F, Aranega AE and Franco D: Identification of regulatory elements directing miR-23a-miR-27a-miR-24-2 transcriptional regulation in response to muscle hypertrophic stimuli. Biochim Biophys Acta 1839: 885-897, 2014.

7. Wang GK, Zhu JQ, Zhang JT, Li Q, Li Y, He J, Qin YW and Jing Q: Circulating microRNA: A novel potential biomarker for early diagnosis of acute myocardial infarction in humans. Eur Heart J 31: 659-666, 2010.

8. Oliveira-Carvalho V, Carvalho VO, Silva MM, Guimarães GV and Bocchi EA: MicroRNAs: A new paradigm in the treatment and diagnosis of heart failure? Arq Bras Cardiol 98: 362-369, 2012.

9. Li H, Zheng D, Zhang B, Liu L, Ou J, Chen W, Xiong S, Gu Y and Yang J: miR-208 promotes cell proliferation by repressing SOX6 expression in human esophageal squamous cell carcinoma. J Transl Med 12: 196, 2014

10. Lee KE, Seo J, Shin J, Ji EH, Roh J, Kim JY, Sun W, Muhr J, Lee S and Kim J: Positive feedback loop between Sox 2 and Sox6 inhibits neuronal differentiation in the developing central nervous system. Proc Natl Acad Sci USA 111: 2794-2799, 2014.

11. Scott O, Pugh J, Kiddoo D, Sonnenberg LK, Bamforth S and Goez HR: Global developmental delay, progressive relapsing-remitting parkinsonism and spinal syrinx in a child with SOX6 mutation. J Child Neurol 29: NP164-NP167, 2014.

12. Prasad V, Lorenz JN, Lasko VM, et al: Ablation of plasma membrane $\mathrm{Ca}^{2+}$-ATPase isoform 4 prevents development of hypertrophy in a model of hypertrophic cardiomyopathy. J Mol Cell Cardiol 77: 53-63, 2014

13. Liao XH, Wang N, Zhao DW, Zheng DL, Zheng L, Xing WJ, Zhou H, Cao DS and Zhang TC: NF-кB (p65) negatively regulates myocardin-induced cardiomyocyte hypertrophy through multiple mechanisms. Cell Signal 26: 2738-2748, 2014.

14. You HW, Chen X, You HJ, Zhang YQ, Cai Y and Liu GZ: Role of endothelin-1 and its receptors on hypertrophy or proliferation of cultured cardial cells. Zhongguo Yi Xue Ke Xue Yuan Xue Bao 28: 520-523, 2006 (In Chinese)

15. Wu Y, Ma XJ, Wang HJ, Li WC, Chen L, Ma D and Huang GY: Expression of $\mathrm{Cx} 43$-related microRNAs in patients with tetralogy of Fallot. World J Pediatr 10: 138-144, 2014.

16. Feng HJ, Ouyang W, Liu JH, Sun YG, Hu R, Huang LH, Xian JL, Jing CF and Zhou MJ: Global microRNA profiles and signaling pathways in the development of cardiac hypertrophy. Braz J Med Biol Res 47: 361-368, 2014.

17. Huang J, Sun W, Huang H, Ye J1, Pan W, Zhong Y, Cheng C, You X, Liu B, Xiong L and Liu S: miR-34a modulates angiotensin II-induced myocardial hypertrophy by direct inhibition of ATG9A expression and autophagic activity. PLoS One 9: e94382, 2014. 